\title{
Author Index Vol. 34, 1996
}

Achiron, A. 63 Aderibigbe, Y.A. 192 Allain, H. 117 Amoretti, G. 36 Ashbrook, D.W. 106 Atobe, M. 82

Barber,Y. 63 Bentue-Ferrer, D. 117 Boiler, F. 76 Bossuyt, A. 175 Brambilla, F. 18 Bujas, M. 201

Caille, P. 98 Caldwell, M.A. 117 Campana, A. 14 Cassano, G.B. 9, 184 Cenacchi, T. 18 Clementz, B.A. 67 Cluydts, R. 175 Conca, A. 204 Cooke, R.G. 26

Corona, G.L. 36 Cucchi, M.L. 36 Curet, O. 98

Dago, K.T. 98 Davis, R.A. 208 DelГOsso, L. 9 Dellu, F. 136 De Meirleir, K. 175 Demets, K. 175 D’Haenen, H. 175 Driscoll, P. 130 Duchêne, J. 166 Durieu, G. 98

Engelsmann, F. 113

Fischhof, P.K. 29 Fischler, B. 175 
Fohanno, D. 76 Frattini, P. 36 Friedmann, A. 29

Gambini, O. 14 Garvey, MJ. 188 Gee, K.W. 106 Gehde, E. 49 Gemignani, A. 184 Giannaccini, G. 9, 184 Granier, L.A. 98 Gureje, O. 192 Guy, T.D. 208

Heinze, H.-J. 49 Hennig, J. 155 Herrmann, W.M. 29 Hüppe, M. 90

Isono, H. 57 Iwanami, A. 57

Jakovljevic, M. 201 Jobert, M. 106,166 Joffe, R.T. 26 Johannes, S. 49

Kagaya, A. 22 Kamijima, K. 57 Kanamori, R. 57 Kariya, T. 82 Kaufman, L. 175 Kerkhofs, M. 1 Klinteberg, B. af 146 König, P. 204 Koppi, St. 204 Kranda, K. 166 Krecke, N. 204 Krijzer, F. 44 Kubota, M. 82 Kusalic, M. 113 
Lanquart, J.-P. 1 Laor, N. 63 Lecrubier, Y. 76 Le Moal, M. 136 Leonard, B.E. 117 Levitt,|AJ. 26 Linkowski, P. 1 Ljubic-Thibal, V. 201 Lucacchini, A. 9, 184 Luthringer, R. 98

Macciardi, F. 14 McDowell, J.E. 67 Macher, J.P. 98 Maggioni, M. 18 Marazziti, D. 9, 184 Martini, C. 9 Mayo, W. 136 Mendlewicz, J. 1 Michiels, V. 175 Milanfranchi, A. 184 Möslinger-Gehmayr, R. 29 Mück-Šeler, D. 201 Münte, T.F. 49

Nakagawara, M. 82 Netter, P. 155 Neuman, E. 76 Nidermaier, B. 90 Nordin, C. 44 Noy, S. 63

Okajima, Y. 57

Panerai, A.E. 18 Patat, A. 98 Pestel, G. 90 Pfanner, C, 184 Piazza, P.V. 136 Preda, S. 36 Presta, S. 184 Pritchard, W.S. 208 
Rancurel, G. 76 Reymann, J.-M. 117 Rinaudo, G. 98 Robb, J.C. 26 Robinson, J.H. 208 Roed, I.S. 155 Rossi, A. 184 Russmann, D.L. 29

Sacerdote, P. 18 Santagostino, G. 36 Scarone, S. 14 Schulz, H. 106 Seewald, M. 49 Siegel, J. 130 Simon, H. 136 Simoncini, M. 9 Singh, V.K. 72 Stanus, E. 1 Stiles, M.F. 208 Swoboda, E. 204

Toren, P. 63 Toussaint, M. 98 Tuason, V.B. 188

Uchitomi, Y. 22 Uhlig, T. 90

Warren, R.P. 72 Weizman, R. 63 Wolmer, L. 63

Yamaji, T. 22 Yamawaki, S. 22 Yokata, N. 22 Young, L.T. 26

Zerbi, F. 36 Zuckerman, M. 125

222 